\title{
EchoGéo
}

$50 \mid 2019$

Varia

\section{Les jardins familiaux franciliens entre urbanisation, végétalisation et agrarisation}

Des espaces au cœur de la fabrique de la ville

\section{Camille Robert-Boeuf}

\section{CpenEdition}

\section{Journals}

Édition électronique

URL : https://journals.openedition.org/echogeo/18288

DOI : $10.4000 /$ echogeo.18288

ISSN : 1963-1197

Éditeur

Pôle de recherche pour l'organisation et la diffusion de l'information géographique (CNRS UMR 8586)

\section{Référence électronique}

Camille Robert-Boeuf, « Les jardins familiaux franciliens entre urbanisation, végétalisation et agrarisation », EchoGéo [En ligne], 50 | 2019, mis en ligne le 31 décembre 2019, consulté le 01 août 2021. URL : http://journals.openedition.org/echogeo/18288; DOI : https://doi.org/10.4000/echogeo. 18288

Ce document a été généré automatiquement le 1 août 2021

EchoGéo est mis à disposition selon les termes de la licence Creative Commons Attribution - Pas d'Utilisation Commerciale - Pas de Modification 4.0 International (CC BY-NC-ND) 


\title{
Les jardins familiaux franciliens entre urbanisation, végétalisation et agrarisation
}

Des espaces au cœur de la fabrique de la ville

\author{
Camille Robert-Boeuf
}

\section{Introduction}

1 Les jardins collectifs constituent une des entrées des études portant sur l'agriculture urbaine en sciences sociales tant ils soulèvent de nombreux enjeux, urbains et nourriciers (Allemand et al., 2016; Aubry, 2015). Le jardinage urbain pose en effet la question des relations que tisse l'individu avec la terre et son environnement (Pourias, 2014 ; Riboulot-Chetrit, 2016), mais aussi celles des sociabilités entre jardiniers, à michemin entre l'entre-soi et le lien social (Cérézuelle, 2003 ; Demailly, 2014 ; Mestdagh, 2018). Forme particulière de l'agriculture urbaine, il est devenu un élément central de la réflexion sur la ville durable et l'aménagement des grandes villes (Aubry t Pourias, 2015 ; Demailly, 2017). Il souligne l'importance des espaces ouverts dans l'espace urbain et, surtout, soulève la question de l'approvisionnement alimentaire des villes: l'agriculture n'est plus seulement un espace de frange, mais devient une véritable « infrastructure de la ville durable " et nourricière (Granchamp-Florentino, 2012, p. 140).

2 Dans ce contexte scientifique, les jardins familiaux, anciennement appelés jardins ouvriers, qui sont un des plus anciens types de jardinage en Europe, ont principalement été étudiés par des historiens et sociologues (Dubost, 1997; Guyon, 2008; Pierson et Cabedoce, 1996; Weber, 1998). Ils ont été notamment présentés comme des espaces domestiques pour des jardiniers, d'origine ouvrière ou issus de catégories sociales modestes, à la fois «chez-soi de plein air » (Weber, 1998, p. 51) et lieu de productions vivrières afin d'améliorer son quotidien alimentaire (Dubost, 1997 ; Pluvinage et Weber, 1992). Rattaché à une réflexion plus large sur la création des banlieues, de l'habitat 
populaire et l'étalement urbain, le jardin familial participe de l'étude des évolutions urbaines (Consales, 2003; Dubost, 1997). Plus récemment, il est convoqué comme facteur de lien social et de citoyenneté dans une ville qui voit la multiplication des formes de jardinage, des jardins partagés aux jardins d'insertion en passant par les potagers pédagogiques (Baudelet et al., 2008 ; Consalès, 2018 ; Guyon, 2004). Ces jardins plus récents ont donné lieu à des travaux sur l'amélioration du cadre de vie, le maintien ou le développement de la biodiversité au sein de la ville, et plus globalement sur le bien-être (Menozzi, 2014 ; Riboulot-Chetrit, 2015). Malgré l'essor des publications sur le sujet, le jardin familial traditionnel est moins traité aujourd'hui que les jardins partagés ou les potagers pédagogiques (Menozzi, 2014), appelant donc à une nouvelle analyse de cet espace au regard de son intégration dans la ville aujourd'hui et de sa mise en concurrence ou en complémentarité avec les autres formes de jardinage.

3 Les jardins familiaux ont relativement peu changé depuis leur apparition au début du XXe siècle : ce sont toujours des regroupements de parcelles individuelles, constitués en association, où un citadin et sa famille pratiquent le jardinage pour leur alimentation et pour leur loisir. Aujourd'hui, selon mes enquêtes, ils sont cultivés par des populations de classes moyennes ou relativement pauvres, mais pas en grande précarité1. Leur maintien dans les grandes villes pendant plus d'un siècle a encouragé une lecture figée et pittoresque de ces espaces (Weber, 1998), ce qui a conduit à les considérer comme des patrimoines paysagers (Cabedoce, 2018 ; Dubost, 2018). Mais audelà, il convient de s'interroger sur leur participation à la fabrique de la ville et leur relatif maintien dans un "urbain généralisé » (Choay, 1994) où les espaces ouverts s'amenuisent. En effet, les jardins familiaux font partie intégrante de la construction de la ville, car ils sont organisés autour de réseaux d'acteurs urbains et participent tant à son urbanisation qu'à sa végétalisation et à son agrarisation. Ce dernier concept, relativement récent dans la littérature scientifique, est compris ici comme un processus qui favorise d'une part l'apprentissage et l'entretien des savoir-faire agricoles chez les populations urbaines; d'autre part le maintien et le développement du foncier agricole en ville - foncier où est exercée une activité agricole professionnelle ou non (Ernwein et Salomon-Cavin, 2014 ; Salomon Cavin et Niwa, 2011). L'agrarisation se distingue de la végétalisation qui désigne, quant à elle, la réintroduction volontaire d'espaces verts et naturels dans la ville.

4 Mon propos est de questionner la spécificité de cette forme de jardinage urbain par rapport aux autres formes de jardins, plus récentes, et d'analyser la structure et le rôle de ces espaces à l'échelle de l'aire urbaine. Le premier temps sera consacré à l'analyse des principaux réseaux d'acteurs autour des jardins, constitués d'acteurs multiples et multiscalaires qui leur confèrent un rôle dans l'aménagement de la ville. Dans un deuxième temps, ces espaces de jardinage seront analysés au prisme du processus d'urbanisation pour mettre en exergue les liens complexes qui les unissent. Enfin, le dernier temps de cet article s'attachera à questionner la participation de ces jardins à un processus d'agrarisation de la ville, bien qu'ils soient souvent perçus comme outil de végétalisation.

5 L'étude des jardins franciliens a été réalisée dans le cadre de mon master puis de ma thèse, de 2013 à 2019. Les enquêtes se sont, dans un premier temps, concentrées sur les jardins familiaux de Stains, de Villejuif et Ris-Orangis pour s'étendre ensuite aux sites de Mantes-la-Ville, Melun, Nanterre et Versailles avec des visites complémentaires à Créteil, Epône ou Crosnes. La multiplicité des sites, leur diversité tant au niveau de leur 
structure associative que de leur localisation - en proche ou lointaine banlieue de Paris - ont facilité la prise en compte des différentes réalités que recouvrent les jardins familiaux en Île-de-France.

6 Le corpus d'enquêtes est essentiellement qualitatif avec des entretiens semi-directifs et de type récit de vie auprès des jardiniers ; l'observation, parfois participante, a en outre été mobilisée. Dix jardiniers ont notamment été suivis sur le long terme (de six mois à plusieurs années) avec des entretiens réguliers qui ont permis d'appréhender leur quotidien, l'évolution des parcelles, et de réaliser de nombreux temps d'observation. Des entretiens ponctuels avec une trentaine de jardiniers et des parcours commentés dans plusieurs jardins familiaux avec un jardinier ou le président de l'association ont complété l'ensemble. Afin de resituer ces enquêtes qualitatives dans un contexte plus large, cent questionnaires ont été distribués aux jardiniers sur les différents terrains étudiés. À cette collecte auprès des jardiniers, s'ajoutent des entretiens avec d'autres acteurs - responsables de mairies, de services techniques, agents de départements, associations régionales de jardins familiaux, et agriculteurs. Un recensement, enfin, a été fait à partir de différentes bases de données existantes ${ }^{2}$ et de géolocalisations par photographies satellites afin de cartographier l'ensemble des jardins franciliens.

\section{Le jardin dans la métropole : un espace structuré à plusieurs échelles}

7 Si le jardinage urbain au sens large est souvent analysé comme une activité qui favorise les sociabilités et le lien social pour les populations jardinières, parfois même à l'échelle du quartier (Alaimo et al., 2010 ; Guyon, 2008), les jardins familiaux instaurent aussi des relations entre différents types d'acteurs à l'échelle métropolitaine, dépassant largement les frontières des parcelles individuelles.

Les jardins familiaux sont une des plus anciennes formes de jardinage urbain encore existantes en France. Les premiers jardins ouvriers (aussi appelés œuvres de charité, puis jardins familiaux à partir de 1952) ont été créés à l'initiative de l'abbé Lemire à la fin du XIXe siècle, puisque ce dernier a participé à un mouvement européen de promotion des lopins individuels pour les populations ouvrières et urbaines pauvres, dont l'office International des Jardins Familiaux se fait l'écho encore aujourd'hui (Pierson et Cabedoce, 1996). En Île-de-France, les jardins familiaux sont estimés au nombre de 594 en 2018, ce qui représente $55,8 \%$ des jardins collectifs recensés par l'IAU ${ }^{3}$. Absents de Paris, ils se trouvent essentiellement en petite couronne, le long des principaux axes de communication franciliens et dans les pôles urbains situés en périphérie (illustration 1 ). Si leur nombre a été très important au début du XXe siècle et pendant l'entre-deux-guerres, beaucoup de parcelles ont disparu à partir des trente glorieuses, car l'amélioration des conditions de vie a rendu leur fonction essentiellement alimentaire - obsolète ${ }^{4}$. Ils connaissent un nouvel essor dans les années 1990 et affirment d'autres fonctions comme leur aspect environnemental et social (Consales, 2000 ; Prédine, 1998). Cette nouvelle période de développement va de pair avec un fort ancrage des jardins à l'échelle régionale et communale, contrairement à la première partie du XXe siècle où les politiques étaient plutôt nationales. 


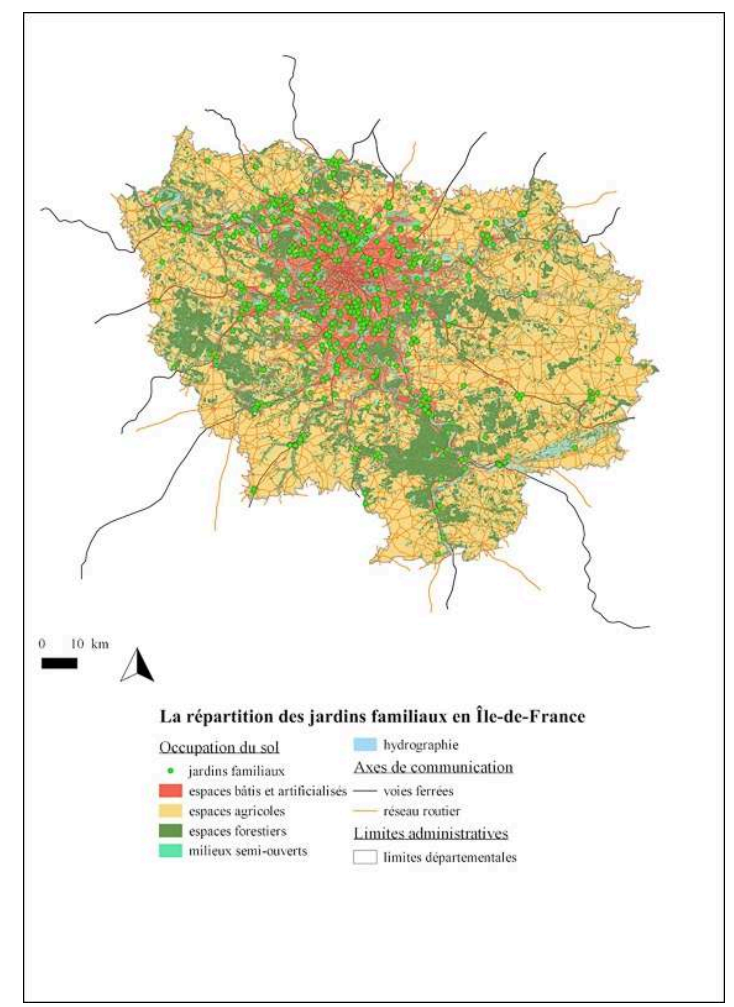

Cette carte a été réalisée grâce aux données de l'IAU de 2013 qui a effectué, dans le cadre du programme JASSUR, un recensement de l'ensemble des jardins collectifs en île-de-France. La collecte de l'IAU s'est faite grâce à la reprise d'un poste du MOS intitulé « jardins familiaux » auquel ont été ajoutées des données fournies par l'analyse de photographies aériennes et des informations issues des associations de jardinage ou d'autres instances locales ${ }^{5}$. Parmi ces données, je n'ai sélectionné que les jardins familiaux et j'ai actualisé les données en consultant des photographies satellites et en croisant les résultats avec ceux de l'Agence régionale de la biodiversité en Île-de-France et de l'Observatoire de l'Agriculture Urbaine en Île-de-France. Le reste de l'occupation du sol correspond au MOS de 2017 élaboré par l'IAU.

Sources : IAU-MOS 2017, IAU 2013. Auteur : (c) 2019, QGIS.

9 À l'origine, les jardins familiaux ont été encadrés par une série de lois édictées par l'État afin de les protéger et d'en encourager la création, mais aussi de contrôler les pratiques jardinières. Ainsi, entre 1912 et 1933, plusieurs lois facilitent les prêts et l'accès au foncier pour les personnes qui désirent créer des jardins et offrent des aides matérielles pour l'achat d'outils ou de graines (Ouzilleau et Chagnot-Loraine, 1999; Pierson et Cabedoce, 1996; Weber, 1998). À partir de 1941, le statut des jardins familiaux se fait plus restrictif avec l'obligation pour tous les jardins de s'organiser en association de type loi 1901 ; surtout, les pratiques agricoles sont définies comme non professionnelles et la vente des productions est interdite.

10 À une législation nationale se substitue aujourd'hui une gestion régionale et locale des jardins familiaux. Ils sont maintenant principalement gérés par des associations en contrat avec des acteurs publics (élus et services techniques). Les associations sont notamment responsables de la gestion quotidienne et sont en grande partie organisées autour du président qui contrôle le bon respect des règlements, l'attribution des parcelles qui se libèrent, et la collecte des cotisations. Elles tissent très souvent des liens avec d'autres associations d'une même commune, pour mener ensemble des activités de sensibilisation à l'environnement, à la défense des espaces verts, etc. Les 
jardins familiaux du parc départemental du Chemin de l'île de Nanterre accueillent ainsi deux autres associations de la commune - l'une à destination des populations handicapées et l'autre qui anime l'un des quartiers populaires de la ville.

11 En Île-de-France, les jardins sont majoritairement installés sur du foncier public et, selon mes terrains, les acteurs privés sont encore aujourd'hui relativement marginaux malgré leur part prépondérante dans la fabrique de la ville. Le foncier des jardins est avant tout communal ou départemental, et parfois régional avec l'Agence des Espaces Verts $^{6}$ (AEV) d'après les données statistiques disponibles ${ }^{7}$. Ces acteurs publics proposent des baux et des règlements corédigés par le service des espaces verts de la ville aux structures associatives en charge de la gestion des parcelles. Leur statut de propriétaire foncier leur donne un droit de regard sur les activités de jardinage : cela se traduit par des exigences de propreté de la part des mairies, d'accessibilité et de visibilité de l'extérieur. Les services techniques servent alors de relais entre élus et jardiniers et surveillent le bon fonctionnement de ces espaces. L'échelle communale s'impose donc comme un niveau majeur dans l'organisation des jardins familiaux.

À plus petite échelle, les jardins familiaux sont souvent réunis dans une fédération qui donne une meilleure lisibilité à ces espaces dans la ville. Ce sont des fédérations nationales pour l'essentiel comme en Île-de-France avec la Fédération Nationale des Jardins Familiaux et Collectifs (FNJFC) et Jardinot. La première est l'héritière de la Ligue du Coin de Terre et du Foyer, créé par l'abbé Lemire en 1896. Jardinot, historiquement issue du regroupement en association de salariés de la SNCF afin d'acheter à prix réduit des fournitures pour le jardinage, regroupe de nombreux jardins familiaux situés sur du foncier de la SNCF, mais s'est ouvert, depuis quelques années, à des jardiniers non cheminots. Si ces deux fédérations nationales rassemblent un nombre important d'associations locales de jardins familiaux, elles ont également en gestion directe certains jardins. Du fait de leur dimension nationale, elles peuvent drainer des capitaux plus importants puisqu'elles récoltent les cotisations de l'ensemble des jardiniers, celles-ci allant financer ensuite les travaux et la rénovation de jardins à l'échelle locale. Elles sont divisées en filiales régionales qui s'occupent de la gestion quotidienne des parcelles et de faire remonter les besoins des jardiniers. Cette structuration multiscalaire des fédérations s'appuie sur une part importante de bénévolat de manière à réduire les dépenses internes qui ne doivent pas venir grever un budget global somme toute faible ${ }^{8}$.

13 Si ces fédérations ne représentent pas l'ensemble des jardins familiaux franciliens, elles donnent une légitimité à ces espaces dans la ville face aux acteurs publics. Elles permettent une reconnaissance de l'utilité des jardins dans un espace urbanisé francilien dense : la FNJFC est, par exemple, reconnue d'utilité publique depuis 1909 et organisme de protection de la nature par la loi du 10 juillet 1976. Les deux fédérations produisent également des chartes ou organisent des concours à l'échelle nationale qui valorisent les fonctions sociale et environnementale du jardin, afin de répondre à différentes exigences des acteurs publics. Ainsi, elles participent chaque année au concours «jardiner autrement», initié par la Société d'Horticulture Nationale Française. De cette manière, la FNJFC et Jardinot créent un contexte favorable pour les jardins familiaux dans la ville et servent d'intermédiaire entre les acteurs publics municipaux, régionaux et même nationaux, en répondant à leurs demandes, et les jardiniers dont elles sont les représentantes. 

permis la transition du foncier maraîcher en lotissements pavillonnaires (Fourcaut, 2000 ; Weber, 1998). Cette évolution s'est accompagnée, dans certains cas, d'un changement de type de populations, puisque les jardins familiaux ont été remplacés, dans la deuxième moitié du XXe siècle, par des lotissements pavillonnaires pour classe moyenne ou aisée (Weber, 1998).

18 À la fin du XXe siècle et au début du XXIe siècle, les jardins familiaux sont réintégrés dans l'aménagement urbain. Sur les communes étudiées, ils deviennent, au vu de leur localisation, des outils de contrôle des friches et des pratiques qui s'y déroulent. La majorité des jardins franciliens pris en compte dans mon analyse sont situés à l'écart du tissu urbain, notamment sur du foncier inondable, inscrit comme espace naturel et non constructible dans les Plans Locaux d'Urbanisme (PLU). Ils sont souvent peu accessibles, relativement loin des transports en commun et les jardiniers utilisent la voiture pour s'y rendre : 80 jardiniers enquêtés sur 100 habitent à moins de $5 \mathrm{~km}$ de leur parcelle et 73 prennent leur voiture, car c'est le moyen de transport le plus pratique quand il faut porter du matériel. Les jardins familiaux du Parc départemental du Chemin de l'île sont une bonne illustration de ces différents éléments : comme leur nom l'indique, ils sont situés à l'intérieur d'un parc et sont accessibles uniquement par l'entrée au sud-ouest de ce dernier. Le parc départemental est, lui-même, peu accessible par rapport aux autres quartiers de la ville, car il est entouré par une zone de friches constituée d'espaces de travaux, d'une ancienne papeterie en rénovation et de la maison d'arrêt des Hauts-de-Seine. Les jardiniers rencontrent donc des problèmes quand ils sont chargés de matériels ou de récoltes comme en témoigne une jardinière : « [je viens en] en voiture. Parce qu'en fait ici on est au nord de Nanterre [...]. Donc venir 
ce n'est pas un problème. Mais alors repartir quand on a une journée de jardinage dans les pattes plus la récolte à rapporter... Et puis honnêtement de chez moi ce n'est pas du tout viable donc je suis obligée de passer par le centre de Nanterre ». Cet espace de frange, à l'écart du reste de la commune, a historiquement favorisé le développement d'activités informelles : s'y est installée une partie du bidonville de Nanterre dans les années 1960 ; certains anciens employés de la papeterie y ont créé des potagers; et la proximité de la maison d'arrêt a fait augmenter les trafics illégaux entre la prison et son environnement direct. Lorsque le parc a été aménagé en 2006, les parcelles de potagers informelles ont été maintenues et réorganisées, et de nouveaux jardiniers se sont installés. Cela a permis d'entretenir une présence régulière dans cet espace et a contribué, selon les jardiniers, à faire baisser les trafics illégaux autour de la prison. Cet exemple montre bien que les jardins familiaux sont utilisés par les acteurs publics pour aménager des zones à l'écart auparavant peu exploitées ou de manière informelle afin d'en régulariser les pratiques puisqu'ils supposent la présence régulière de populations jardinières encadrées par des associations.

Plus récemment encore, les jardins familiaux sont fortement intégrés dans des projets urbains pour aménager les interstices d'espaces ouverts dans un contexte d'artificialisation des terres et de densification du bâti. Les aménageurs publics favorisent, voire imposent, en effet l'implantation de jardins familiaux dans les nouvelles Zones d'Aménagement Concerté (ZAC) ou quartiers d'habitations: c'est notamment le cas dans trois des quatre communes étudiées (Nanterre, Melun et Versailles). Néanmoins, les jardins y sont souvent plus réduits avec de plus petites parcelles, tant la pression foncière est forte: quand les jardins familiaux les plus anciens comptaient plus d'une centaine de parcelles de 200 ou $300 \mathrm{~m}^{2}$, les nouveaux n'en regroupent que quelques dizaines de $100 \mathrm{~m}^{2}$ maximum. À cette réduction des parcelles s'ajoute une mutualisation des abris conçus plutôt comme des lieux de stockage du matériel alors qu'ils faisaient office de véritable petite maison au début du XXe siècle. Les jardins familiaux sont ainsi devenus partie prenante des projets d'aménagement : de plus en plus imposés par les normes urbaines durables qui doivent dessiner une trame verte, ils participent à une nouvelle densification du bâti.

Cette évolution a pour conséquence un déplacement des jardins familiaux à l'échelle métropolitaine : alors qu'ils sont traditionnellement très implantés en petite couronne, les jardins les plus récents sont aujourd'hui majoritairement installés dans le périurbain francilien. Dans les nouveaux projets d'aménagement de la banlieue parisienne, cette forme de jardinage est progressivement délaissée au profit des jardins partagés ou des potagers pédagogiques. Si les aspects fonciers en sont une clef d'explication, les nouvelles fonctions assignées aux jardins y participent tout autant. Les jardins familiaux nourriciers appellent en effet des lopins relativement grands pour un nombre limité de personnes quand l'entretien collectif dans les jardins partagés permet d'accueillir un nombre d'allocataires plus important sur des superficies moindres. Surtout, les collectivités locales plébiscitent les fonctions non vivrières : à Nanterre, si la mairie ne souhaite pas fermer les jardins familiaux de la commune, elle encourage plutôt aujourd'hui les autres formes de jardinage qui mettent l'accent sur la sensibilisation à l'environnement et non sur la production agricole, et favorise donc l'installation d'autres types de jardins. Une responsable du service Biodiversité de la commune constate: «La mandature actuelle c'était plutôt développer les jardins partagés. $Y$ en avait un au départ qui est au mont Valérien qui est géré par l'association C si Bio, et qui avait répondu à un appel à projets. Et depuis je crois qu'on a cinq ou six 
structures qui gèrent des jardins partagés. Donc nous la politique de la direction de l'environnement, c'est de les accompagnés techniquement [...] Et on travaille aussi sur l'agriculture urbaine, sur le volet potager avec les écoles : il y a à peu près cinquante groupes scolaires sur Nanterre [...] et il y a 45 potagers en service actuellement. »

21 Les jardins familiaux ont donc influencé l'évolution urbaine de la région francilienne en participant à l'urbanisation des périphéries de Paris et notamment de la petite couronne au cours du XXe siècle. Ces dernières années, la pression foncière remet en cause leur intégration dans la ville dense, d'autant que d'autres jardins, partagés ou pédagogiques, s'accommodent mieux des contraintes foncières et viennent plus facilement répondre aux nouvelles demandes des acteurs publics, demandes sociales plus que nourricières qui les intègrent toujours plus à la catégorie d'espaces verts.

\section{Agrarisation vs végétalisation : le rôle des jardins familiaux disputé dans la ville}

Les jardins familiaux sont historiquement des espaces nourriciers pour les associations qui les entretiennent et cette fonction agricole reste importante encore aujourd'hui pour les jardiniers. Toutefois, leur maintien à l'échelle régionale est avant tout dû à leur potentiel naturel et environnemental souligné par les acteurs publics et les documents d'urbanisme.

23 La fonction première du jardin familial est son aspect vivrier, car les autres fonctions, de loisir, sociales ou environnementales, arrivent en second lieu et sont subordonnées à la production agricole. La majorité des associations de jardinage continue d'ailleurs d'imposer la culture de plantes vivrières sur au moins $50 \%$ de la parcelle allouée. Quant aux jardiniers, les avis sont unanimes. Sur 100 jardiniers interrogés, 70 disent qu'ils jardinent pour avoir accès à des produits biologiques et 62 estiment avoir modifié leurs pratiques alimentaires depuis qu'ils ont accès à un jardin : ils mangent plus de légumes variés et prêtent tout particulièrement attention à la saisonnalité des produits (pour 86 d'entre eux). Lors des entretiens, beaucoup ont affirmé faire des économies et réduire leurs dépenses alimentaires de juin à septembre, lors de la période de récolte. En outre, une grande partie des jardiniers rencontrés transforment ses récoltes (44 enquêtés sur 100 suivant les questionnaires), ce qui allonge la période d'apport alimentaire jardinier qui reste pourtant minoritaire. L'apport alimentaire ne concerne pas d'ailleurs seulement celui qui entretient une parcelle puisque les dons sont fréquents : 77 enquêtés donnent une partie de leur production à des membres de la famille (souvent aux enfants et petits-enfants), 79 en donnent à leurs amis et beaucoup affirment, dans les entretiens, cultiver en fonction des goûts de leurs conjoints et enfants.

Espaces vivriers, ils favorisent la transmission et même le développement de pratiques agricoles. S'ils ont souvent un savoir-faire initial - 69 enquêtés ont appris avec leurs parents et/ou grands-parents -, les jardiniers apprennent énormément par la pratique et au contact de leurs voisins, certains, plus expérimentés que d'autres, étant érigés en référence. Telle jardinière de Versailles constate: "J'en donne beaucoup, moi [des conseils], j'en donne beaucoup, et puis des fois on me dit à moi : "on peut semer ça?", j'ai dit "t'as vu la lune ?". Parce que la lune joue, les paysans ils regardent la lune. Et puis je dis: "écoute tu vois bien qu'il a fait froid toute la semaine, on ne peut rien planter [...]" ». Ces pratiques agricoles héritées, sans cesse améliorées par 
l'apprentissage, s'inventent aussi dans les liens que tissent les jardiniers avec différents acteurs des périphéries rurales : il en est ainsi des centres équestres ou des agriculteurs proches afin de récupérer du fumier gratuitement ou relativement peu cher. Des relations de confiance et des sociabilités se dessinent alors aux marges de la ville qui viennent élargir le territoire jardinier aux campagnes proches.

L'activité agricole continue donc de structurer fortement l'organisation quotidienne des jardins familiaux et y demeure essentielle, comparé aux formes de jardinage urbain plus récentes. Ils affirment ainsi leurs spécificités par rapport aux autres espaces verts et contribuent à diffuser, auprès des populations urbaines, des savoir-faire agricoles. En ce sens, le jardin familial peut être compris comme un espace qui favorise l'agrarisation de la ville, telle que définie en introduction (Ernwein et Salomon-Cavin, 2014 ; Janin, 2018 ; Vidal et Fleury, 2009). Cette caractéristique explique l'engouement des individus pour ce type de jardins : les jardiniers rencontrés sont attirés par le travail de la terre, le contact avec celle-ci et la production de leur propre nourriture. Les liens avec la terre et le cycle des plantes sont les motivations les plus avancées en entretien, comme l'explique ce jardinier de Melun: «Ah oui, j'adore le jardin. J'adore la terre moi de toute façon... il faut que je touche la terre. [...] Pour moi, c'est très important [le jardinage], je dirais même que je ne peux pas vivre sans, j'ai besoin de me recueillir avec la nature et ça pour moi c'est important. Si je peux savoir encore plus de choses, c'est encore mieux. [...] Moi j'aime bien jardiner c'est sûr, et j'aime bien me retrouver dans la nature, j'ai besoin d'avoir le contact [...] Et puis bon, c'est une passion. »

Néanmoins, cette fonction agricole n'est pas pleinement comprise ou assumée par les acteurs publics, puisque ces derniers conçoivent les jardins familiaux avant tout comme des espaces verts. En effet, selon eux, cette forme de jardinage urbain présente divers avantages, le premier étant sa capacité à créer et entretenir des espaces verts à moindre coût. Si les services techniques sont largement mobilisés lors de la phase de mise en place, ils laissent ensuite la gestion des parcelles aux citoyens qui "s'autogèrent", comme cela a été dit dans nombre d'entretiens. Cette fonction sans cesse réaffirmée renvoie à une lecture esthétique du jardin et appelle des pratiques agricoles qu'on pourrait qualifier de paysagères : le jardinier doit penser son espace et ses manières de faire dans une vision paysagère et esthétique, ce qui n'est pas sans contrainte. Ainsi que le signale un des employés du Domaine du Château de Versailles, propriétaire d'un des sites des jardins familiaux de Versailles étudiés : "comme on considère que les jardins familiaux participent d'une animation paysagère, on leur impose de plus en plus de contraintes, y compris en termes de gestion quotidienne». Les fédérations de jardins sont amenées à intégrer cette lecture des acteurs publics dans leurs règlements et dans leurs discours, ce qui transforme les jardins familiaux en "vitrines de la nature en ville». Certaines pratiques agricoles sont codifiées, voire interdites: la limitation des récupérateurs d'eau, la pose de filets pour protéger les semis, l'utilisation d'objets de récupérations, l'interdiction de serres trop imposantes et le nettoyage régulier des parcelles, car l'herbe est un signe de non-traitement chimique. Les jardiniers doivent alors adapter, voire cacher, les comportements critiqués: ils rangent leurs parcelles avant la visite des services municipaux ou départementaux, cachent parfois leurs récupérateurs d'eau, aménagent leurs abris lorsque ceux-ci sont trop petits ou peu pratiques. Dans cette perspective, le jardin familial se doit d'être ouvert à un public large et plusieurs associations enquêtées organisent des visites avec les écoles ou réservent des parcelles spécifiques pour des personnes hospitalisées. Par exemple, les jardins familiaux de Chantereine à Mantes-la- 
Ville ont des parcelles surélevées pour les personnes handicapées, une parcelle occupée par une infirmière qui amène ses patients, en collaboration avec l'hôpital de jour, et enfin reçoivent la visite d'élèves d'écoles élémentaires. Les jardins familiaux sont donc considérés comme des espaces verts de la ville, au service d'une sensibilisation à l'environnement des populations urbaines.

Cette lecture au prisme de leur dimension naturelle et environnementale conduit à leur inscription dans les documents d'urbanisme, notamment les Plans d'Aménagement et de Développement Durable (PADD) qui accompagnent les Scot et les trames vertes. Dans trois des quatre communes franciliennes étudiées, les jardins familiaux sont ainsi considérés comme des espaces à conserver voire à développer, avec des élus très impliqués dans une politique forte de création de jardins. C'est le cas à Versailles où la responsable du service des espaces verts explique : «[...] je pense qu'on est une ville extrêmement verte et ça a toujours été la volonté du maire de privilégier les espaces verts. Par exemple sur Sévigné-Charcot, le choix du maire ça a été de dire "je ne densifie pas la population habitable, je crée des jardins". C'est sur du foncier constructible, donc c'est un parti pris très fort. [...] ». Ainsi, dans les communes où le foncier est disponible, souvent en périphérie de la métropole parisienne, le modèle du jardin familial continue d'être attractif du fait de son rôle environnemental : d'après plusieurs entretiens, la FNJFC et Jardinot observent un intérêt croissant des élus locaux pour la création de jardins familiaux, avec des demandes d'accompagnement plus nombreuses ces dernières années. La recherche d'amélioration de l'offre d'espaces verts en ville par certaines communes légitime donc le maintien et la création de jardins familiaux, essentiellement hors de la petite couronne où le foncier est plus accessible. Cette tendance est renforcée dans les communes où le jardin familial fait figure de patrimoine, comme dans le cas de Versailles où certaines associations existent depuis le début du XXe siècle, à l'image des jardins familiaux des Petits Bois à Versailles, créés en 1906, qui possèdent depuis 2014 le label de « jardin remarquable ».

Le foncier des jardins familiaux n'est donc pas reconnu comme un espace agricole dans les PADD ou même les PLU et il peut accueillir d'autres formes d'espaces verts selon la volonté des élus locaux. Cela peut s'expliquer par la valorisation de la fonction environnementale des espaces ouverts en général et du jardinage urbain en particulier dans les politiques publiques locales franciliennes récentes. En effet, les jardins familiaux ne sont pas distingués des autres formes de jardinage et sont souvent réunis, dans les discours et les documents comme le Schéma Directeur de la Région Île-deFrance (SDRIF), sous le terme de "jardins collectifs » : ces espaces sont alors désignés avant tout comme un moyen pour répondre aux impératifs de la ville durable et aux exigences environnementales des urbains. Les jardins familiaux sont donc un lieu de promotion des savoir-faire agricoles, mais n'entrainent pas forcément l'inscription de leur foncier comme foncier agricole et une reconnaissance de leur spécificité auprès des acteurs publics, ce qui nuance en partie leur potentiel d'agrarisation de la ville.

Le jardin familial se démarque des autres formes de jardinage urbain par sa fonction nourricière et, par-là, affirme une activité agricole en ville et légitime sa place dans la métropole. Paradoxalement, il doit son maintien dans la région francilienne grâce à sa capacité à végétaliser la ville et à sa dimension environnementale, lecture soutenue et développée par les acteurs publics et dans les documents d'urbanisme. Au travers de cette tension entre végétalisation et agrarisation, le rôle agricole des jardins familiaux est alors encore relativement fragile, car il reste tributaire des élus locaux. 


\section{Conclusion}

Les jardins familiaux sont une des plus anciennes formes de jardinage urbain en Île-deFrance. Ils se structurent à plusieurs échelles et mettent en relations des associations, des citoyens et des acteurs publics à l'échelle locale et régionale. Répondant à des enjeux à la fois locaux et métropolitains, ils ont participé au processus d'urbanisation, mais aussi de manière quasi paradoxale à une forme d'agrarisation de la ville. S'ils ont servi, originellement, à l'urbanisation de la banlieue parisienne, ils en sont aujourd'hui victimes, puisqu'ils ne répondent pas, ou du moins difficilement, aux impératifs de densification de la ville. Ils proposent cependant une autre manière d'envisager les espaces ouverts de la ville, car ils promeuvent la réintroduction d'espaces vivriers et la diffusion de pratiques agricoles. Face à la démultiplication des fonctions du jardinage dans la ville - fonctions qui touchent au social, à l'environnement ou aux loisirs - les jardins familiaux viennent réinterroger la fonction vivrière, soit les nouvelles relations en train de s'inventer de la ville à l'agriculture et à la terre. Toutefois, cette fonction agricole est relativement fragile, car elle n'est pas entièrement reconnue par les acteurs publics et par les documents d'urbanisme qui les voient plutôt comme un moyen de végétalisation de la ville et les associent aux autres formes de jardinage urbain plus récentes où cet aspect vivrier est moins présent.

\section{BIBLIOGRAPHIE}

Alaimo K., Reischl T.-M., Allen J.-O., 2010. Community gardening, neighborhood meetings, and social capital. Journal of Community Psychology, vol. 38, n 4, p. 497514.

Allemand S., Heurgon E., Pelluchon C., 2016. Nourritures jardinières dans les sociétés urbanisées : Colloque de Cerisy. Paris, France, Hermann, 271 p.

Aubry C., 2015. Les agricultures urbaines et les questionnements de la recherche. Pour, $\mathrm{n}^{\circ} 224$, p. 35-49.

Aubry C., Pourias J., 2015. Agricultures et jardinages urbains : quelles contributions à la ville durable en Europe ? La Revue de l'Académie d'agriculture, $\mathrm{n}^{\circ}$ 5, p. 3236.

Bastié J., 1964. La Croissance de la banlieue parisienne, Paris, Presses universitaires de France, 624 p.

Baudelet L., Basset F., Le Roy A., Clément G., 2008. Jardins partagés : utopie, écologie, conseils pratiques. Mens, France, Terre vivante, $157 \mathrm{p}$.

Cabedoce B., 2018. 1940-1952 : une période charnière pour les jardins ouvriers. In Situ. Revue des patrimoines [En ligne], $\mathrm{n}^{\circ}$ 37. http://journals.openedition.org/insitu/18752 - DOI : 10.4000/insitu. 18752

Cérézuelle D., 2003. Les jardins familiaux, lieux d'initiation à la civilité. Communications, vol. 74, $\mathrm{n}^{\circ} 1$, p. 6583. 
Choay F., 1994. Le règne de l'urbain et la mort de la ville. In Barre F., La Ville, Art et Architecture en Europe 1870-1993. Paris, Ed. C. Georges Pompidou, p. 2635.

Consalès J.-N., 2018. Des jardins ouvriers au jardinage de rue : pour une géohistoire des jardins collectifs à Marseille. In Situ. Revue des patrimoines [En ligne], $\mathrm{n}^{\circ} 37 . \mathrm{http}: / /$ journals.openedition.org/insitu/19252 - DOI : 10.4000/insitu.19252

Consalès J.-N.., 2003. Les jardins familiaux de Marseille, Gênes et Barcelone. Rives méditerranéennes [En ligne], $\mathrm{n}^{\circ}$ 15. http://journals.openedition.org/rives/449 - DOI : 10.4000/rives.449

Consalès J.-N., 2000. Les jardins familiaux marseillais : laboratoires territoriaux d'une agriculture urbaine en Méditerranée. Méditerranée, vol. 95, n 3, p. 81-88.

Demailly K.-E., 2014. Les jardins partagés franciliens, scènes de participation citoyenne ? EchoGéo [En ligne], $\mathrm{n}^{\circ}$ 27. http://journals.openedition.org/echogeo/13702 - DOI : 10.4000/echogeo.13702

Demailly K.-E., 2017. Les jardins partagés franciliens. Des territoires de transition environnementale? Géographie et cultures, n 101, p. 7995.

Dubost F., 1997. Les jardins ordinaires. Paris - Montréal, Éd L’Harmattan, 174 p.

Dubost F., 2018. Jardins collectifs : de l'abbé Lemire aux jardins d'insertion. Typologies Expériences - Enjeux de conservation. In Situ. Revue des patrimoines [En ligne]. $\mathrm{N}^{\circ} 37 . \mathrm{http}: / /$ journals.openedition.org/insitu/19624

Ernwein M., Salomon-Cavin J., 2014. Au-delà de l'agrarisation de la ville : l'agriculture peut-elle être un outil d'aménagement urbain ? Discussion à partir de l'exemple genevois. Géocarrefour, vol. $89, n^{\circ} 12$, p. 3140 .

Fourcaut A., 2000. La banlieue en morceaux : la crise des lotissements défectueux en France dans l'entredeux-guerres. Grâne, France, Créaphis, 339 p.

Granchamp-Florentino L., 2012. L'agriculture urbaine. Un enjeu de la ville durable. Revue des Sciences sociales, $\mathrm{n}^{\circ} 47$, p. 140-152.

Guyon F., 2004. Les jardins familiaux : miroirs des politiques de la cité. Loisir et Société / Society and Leisure, vol. 27, $\mathrm{n}^{\circ} 2$, p. 529546.

Guyon F., 2008. Les jardins familiaux aujourd'hui : des espaces socialement modulés. Espaces et sociétés, vol. 134, n³, p. 131147.

Janin R., 2018. La ville agricole : l'agriculture vit une révolution urbaine sans précédent traduisant un changement de civilisation profond. Fourneaux, Éditions Openfield, $74 \mathrm{p}$.

Menozzi M.-J., 2014. Les jardins dans la ville entre nature et culture. Rennes, France, Presses universitaires de Rennes, $362 \mathrm{p}$.

Mestdagh L., 2018. Jardiner entre soi. Paris, L'Harmattan, 306 p.

Ouzilleau B., Chagnot-Loraine I., 1999. Les jardins familiaux en Île-de-France. Paris, France, IAURIF, $98 \mathrm{p}$.

Pierson P., Cabedoce B., 1996. Cent ans d'histoire des jardins ouvriers : 1896-1996. La ligue française du coin de terre et du foyer. Grane, Creaphis, $221 \mathrm{p}$.

Pluvinage M., Weber F., 1992. Les jardins populaires : pratiques culturales, usages de l'espace, enjeux culturels. Éléments d'histoire et d'ethnographie. Rapport de recherche pour la Mission du Patrimoine ethnologique du Ministère de la Culture. Paris, France, Ministère de la Culture, 208 p. 
Pourias J., 2014. Production alimentaire et pratiques culturales en agriculture urbaine Analyse agronomique de la fonction alimentaire des jardins associatifs urbains à Paris et Montréal (Thèse). Paris, AgroParisTech, $271 \mathrm{p}$.

Prédine É., 1998. Jardins ouvriers : l'art et la manière. Paris, La Maison rustique, 143 p.

Raymond H., Haumont N., Dezès M.-G., Haumont A., 2001. L'habitat pavillonnaire. Paris - Montréal, L'Harmattan, $114 \mathrm{p}$.

Riboulot-Chetrit M., 2015. Les jardins privés : de nouveaux espaces clés pour la gestion de la biodiversité dans les agglomérations? Articulo - Journal of Urban Research [En ligne], $\mathrm{n}^{\circ}$ Special 6. http://journals.openedition.org/articulo/2696 - DOI : 10.4000/articulo.2696

Riboulot-Chetrit M., 2016. Les habitants et leur jardin. Relations au vivant, pratiques de jardinage et biodiversité au cœur de l'agglomération parisienne. Thèse, Paris, Université Paris 1 PanthéonSorbonne.

Salomon Cavin J., Niwa N., 2011. Introduction : Agriculture urbaine en Suisse. Au-delà des paradoxes. Urbia, Les cahiers du développement urbain durable, $\mathrm{n}^{\circ} 12$, p. 5-16.

Vidal R., Fleury A., 2009. Aménager les relations entre la ville et l'agriculture. De nouveaux enjeux territoriaux et une nouvelle approche "agriurbaniste“. Urbia. Les cahiers du développement urbain durable, $\mathrm{n}^{\circ} 8$, p. 127-142.

Weber F., 1998. L'honneur des jardiniers, les potagers dans la France du XXe siècle. Paris, Belin, 287 p.

\section{NOTES}

1. Selon les enquêtes menées, le revenu moyen des jardiniers est juste en dessous du revenu médian francilien. Il faut bien sûr garder à l'esprit que le revenu médian francilien est plus élevé que dans les autres régions françaises et il faudrait d'autres études à l'échelle nationale pour confirmer ces résultats.

2. Notamment celles du Programme JASSUR (en collaboration avec l'IAU), de l'Agence régionale de la biodiversité en Île-de-France et de l'observatoire de l'Agriculture Urbaine en Île-de-France.

3. https://www.iau-idf.fr/fileadmin/NewEtudes/Etude_1461/NR_773_web.pdf

4. Il n'existe pas de recensement historique précis et les chiffres varient selon les sources, cependant, à l'échelle nationale, la Fédération Nationale des Jardins Familiaux et Collectifs, une des principales associations de jardins familiaux, gérait 47000 jardins ouvriers en 1920 contre 15000 aujourd'hui.

5. https://www.iau-idf.fr/fileadmin/NewEtudes/Etude_1461/NR_773_web.pdf

6. Cet établissement public créé en 1976 est chargé de mettre en œuvre la politique environnementale de l'île-de-France et de protéger les espaces verts de l'urbanisation. Il possède $\mathrm{du}$ foncier dans toute la région et a quelques jardins familiaux sur son territoire.

7. Données issues du programme JASSUR, de l'Agence régionale de la biodiversité en Île-deFrance et de l'Observatoire de l'Agriculture Urbaine en Île-de-France.

8. Si leur financement se limite souvent aux cotisations de leurs membres, elles peuvent néanmoins recevoir des aides ponctuelles notamment au moment de la création de nouveaux jardins de la part de la commune ou du département. Jardinot a également une aide du CE de la SNCF et de l'office National de l'Eau et des milieux aquatiques afin d'organiser un concours de jardinage qui favorise les pratiques écologiques. 


\section{RÉSUMÉS}

Les jardins familiaux sont une des formes les plus anciennes du jardinage urbain en France. Ces dernières années, ils entrent en concurrence et en complémentarité avec d'autres formes de jardinage plus récentes comme les jardins partagés ou potagers pédagogiques. En île-de-France, ces jardins familiaux s'inscrivent dans la ville en articulant plusieurs échelles et différents types d'acteurs et révèlent certains enjeux d'aménagement urbain à lire dans la durée. Participant originellement à l'urbanisation des banlieues, ils en sont aujourd'hui les victimes. Cependant, plus qu'une végétalisation de la ville et une amélioration du cadre de vie des urbains comme les autres formes de jardinage, ils favorisent une agrarisation de la métropole aujourd'hui discutée.

Allotment gardens are one of the oldest forms of urban gardening in France. In recent years, they are competing but also complementing other more recent forms of gardening such as community gardens or educational gardens. In Parisian region, these allotment gardens are part of the city because they associate several types of actors on several scales and underline issues of the urban planning. Originally involved in the urbanisation of the suburbs, they are now the victims of this urbanisation. However, more than a revegetation and an improvement of the living environment of the city, they promote a debated agrarisation of the metropolis.

\section{INDEX}

Mots-clés : jardin familial, jardinage urbain, urbanisation, agrarisation, relation ville-campagne Keywords : allotment garden, urban gardening, urbanisation, agrarisation, urban-rural relationship

\section{AUTEUR}

\section{CAMILLE ROBERT-BOEUF}

Camille Robert-Boeuf, kamrb@hotmail.fr, est doctorante en géographie à l'Université ParisNanterre et chercheuse associée à l'Université Fédérale de Kazan (Russie). Elle a récemment publié :

- Robert-Boeuf C., 2017. Cultiver la ville en Russie. La datcha à Kazan : histoire de pratiques jardinières. Géographie et Cultures, $\mathrm{n}^{\circ} 101, \mathrm{p}$. 17-34.

- Robert-Boeuf C., 2017. Les jardins collectifs en Ile-de-France et à Kazan. Des espaces hybrides pour réinterroger les relations villes-campagnes. In Berger M. et Chaléard J.-L. (dir), Villes et campagnes en relations : regards croisés Nords-Suds. Paris, Ed. Karthala, p. 200-212.

- Robert-Boeuf C., 2017. Les jardins collectifs constitutifs de réseaux alternatifs au service des urbains. Étude de cas de l'Ile-de-France et de Kazan (Russie). In Gauvard C. (dir.), Appartenances et pratiques des réseaux. Éditions électroniques du CTHS, p. 205-217. 